Document downloaded from:

http://hdl.handle.net/10251/140955

This paper must be cited as:

Mesejo Conejos, C.; Martinez Fuentes, A.; Reig Valor, C.; Agustí Fonfría, M. (08-2). The flower to fruit transition in Citrus is partially sustained by autonomous carbohydrate synthesis in the ovary. Plant Science. 285:224-229. https://doi.org/10.1016/j.plantsci.2019.05.014

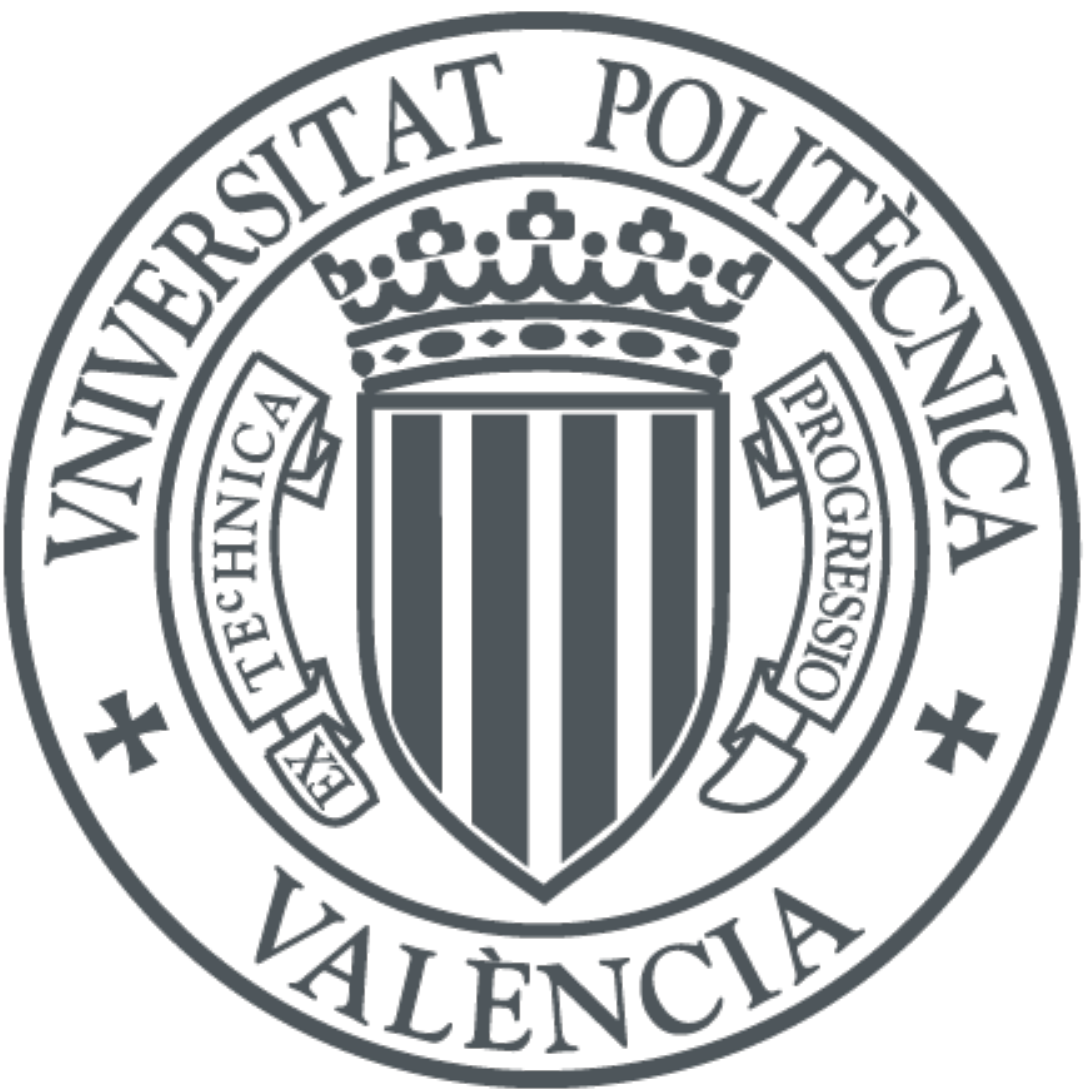

The final publication is available at

https://doi.org/10.1016/j.plantsci.2019.05.014

Copyright Elsevier

Additional Information 


\title{
The flower to fruit transition in Citrus is partially sustained by autonomous carbohydrate synthesis in the ovary
}

\author{
Mesejo C*, Martínez-Fuentes A, Reig C, Agustí M. \\ Instituto Agroforestal Mediterráneo, Universitat Politècnica de València, Camino de \\ Vera, s/n, 46022 Valencia, Spain. \\ *Corresponding author: carmeco@upv.es
}

\begin{abstract}
Why evergreen fruit tree species accumulate starch in the ovary during flower bud differentiation in spring, as deciduous species do during flower bud dormancy, is not fully understood. This is because in evergreen species carbon supply is assured by leaves during flower development. We suggest the existence of an autonomous mechanism in the flowers which counteracts the competition for photoassimilates with new leaves, until they become source organs. Our hypothesis is that starch accumulated during Citrus ovary ontogeny originates from 1) its own photosynthetic capacity and 2) the mobilization of reserves.
\end{abstract}

Through defoliation experiments, we found that ovaries accumulate starch during flower ontogeny using a dual mechanism: 1) the autotrophic route of source organs activating Rubisco $(R b c S)$ genes expression, and 2) the heterotrophic route of sink organs that hydrolyze sucrose in the cytosol. Defoliation 40 days before anthesis did not significantly reduce ovary growth, flower abscission or starch concentration up to 20 days after anthesis (i.e. 60 days later). Control flowers activated the energy depletion signaling system (i.e. SnRK1) and $R b c S$ gene expression around athesis. Defoliation accelerated and boosted both activities, increasing SPS gene expression (sucrose synthesis), and SUS1, SUS3 and cwINV (sucrose hydrolysis) to maintain a glucose threshold which satisfied its need to avoid abscission.

Key words: Citrus, parthenocarpy, starch, sucrose, Rubisco, SnRK1 


\section{Introduction}

Most fruit tree species set flowers in a very low proportion. In particular, it is a common phenomenon of self-incompatible hermaphroditic species to have a low fruit/flower ratio (i.e. fruit set), which limits fruit yield [1]. In general, fruit tree species have three waves of flower/fruit abscission, at 1) pre-anthesis, in trees with a very high flowering intensity, 2) at post-anthesis, and 3) in newly set fruitlets. They vary in length and intensity depending on the species, as well as exogenous and endogenous factors [2].

Physiological flower abscission has been attributed to a shortage of carbohydrate $(\mathrm{CH})$ status in both deciduous and evergreen fruit tree species [3,4], although there are serious doubts regarding this hypothesis [5]. In some temperate deciduous species, such as Prunus sp. or Malus sp, floral bud differentiation occurs in late summer-early autumn before dormancy, whereas blooming takes place in late winter-early spring. Besides, floral buds sprout earlier than do vegetative buds, and hence flower growth from budbreak to early fruiting should not depend solely on the current photosynthesis [6]. Accordingly, the onset of ovary growth has been suggested as an autonomous process that correlates ovary starch depletion until leaves export $\mathrm{CH}[4,6]$.

On the other hand, in evergreen woody species (citrus, avocado, olive, etc.) floral buds do not differentiate before dormancy but rather during budbreak in spring. Thus, blooming and vegetative growth occur simultaneously. Therefore, flowers and new leaves compete for $\mathrm{CH}$ produced by the mobilization of starch reserves and the photosynthates from the mature leaves [7,8]. Given that the sink-to-source transition of young leaves occurs around 4 weeks after full bloom [8], competition for $\mathrm{CH}$ lasts until the beginning of the first fruitlet abscission, with many ovaries on the tree having high rates of cell division and respiration [9]. Despite this, flowers accumulate starch in the ovary during the development of the inflorescence, as observed in citrus [10,11], avocado [12,13] and olive [14]. This content has been related to flower quality [4, 14] and initial ovary growth and retention [12,13].

The fact that this phenomenon occurs in both deciduous and evergreen fruit tree species suggests the existence of an autonomous mechanism of the flowers to counteract the competition with the new leaves [15], allowing the flower-to-fruit transition. Metabolic pathways involved in the starch biosynthesis are different between source organs (mainly leaves) and sink organs (mainly seeds). In source organs, starch 
is directly synthesized as a final product of photosynthesis in the chloroplast by means of the enzyme ADP glucose-pyrophosphorylase (ADPGP) and starch synthase (SS), when there is an excess of triose phosphate. In this case, carbon is derived from fructose-6-P [16-18]. In the case of sink organs, the ADP-glucose needed for starch synthesis is derived from sucrose hydrolysis in the cytosol by the action of sucrose synthase (SUS), and in the apoplast by the action of the cell wall invertase (cwINV) [16, 19].

In spite of this, the mechanism of starch accumulation in the ovary, and its physiological role, is still not fully understood. Flowers and fruits are sink organs, but they are also able to fix carbon temporarily in amounts which are highly variable among species, such as peach [20], apple [21] and grape [22]. Accordingly, we may ask up to what extent the starch accumulated in the ovary walls contributes to the early development of flower/fruit. If starch accumulation is an autonomous mechanism of the flower to facilitate fruit set, the ovary of evergreen trees might accumulate starch to counteract competition for $\mathrm{CH}$ with the leaves, as occurs in deciduous trees. Therefore, we propose the hypothesis that the starch accumulated during ovary ontogeny is crucial to set the fruit, maintaining an adequate level of glucose that temporarily releases the ovary from the competition with young leaves until they are photo-synthetically active. Starch in the ovary originates from 1) its own photosynthetic capacity, and 2) the mobilization of the reserves. To this end, we study the time-course of $\mathrm{CH}$ concentrations in the flowers, the activation of the SnRK1 (SNF1-related kinase 1) sugar deficiency sensor, which is activated by diverse energy-depleting stress conditions and maintains energy homeostasis by modification of key metabolic enzymes [23], and the starch and sucrose synthesis and hydrolysis with regard to flower abscission. We used the Citrus clementina tree as a model system because it is a self-incompatible hermaphroditic species that flowers profusely and sets a very low percentage of fruits, ranging from 0.1 to $10 \%$ depending on the cultivars, and there is a wide body of knowledge about the nutritional regulation of fruit set [3, 8, 10, 24-27].

\section{Materials and Methods}

\subsection{Plant material, experimental design and defoliation treatments}


The experiments involved 15-year-old Citrus clementina trees (cv. Marisol) grafted onto 'Carrizo' citrange (Citrus sinensis x Poncirus trifoliata) rootstock, planted 5 m x 5 m apart in a loamy clay soil, with drip irrigation in the Cooperativa de Llíria Research Orchard (Casinos, Valencia, Spain). Eight trees were selected for the experiments. Of these, 4 trees were only used to study the effect of defoliation on the time-course of flower/fruit development and abscission, and the other 4 trees were only used for tissue sampling so as not to interfere in the fruit abscission rate. Treatments were as follows: two of the four main branches in each tree were fully defoliated and the other two were left as control. Branches were considered as individual replicates given that woody branches fix carbon and are completely autonomous for carbohydrates [28]. Defoliation of mature and young leaves was performed forty days before anthesis, i.e. when new buds (either floral and vegetative) were at their early differentiation stage. The experiment was performed with a minimum of 400 flowers per branch [11], and repeated in two consecutive seasons.

\subsection{Fuit development evaluation and sampling}

Fruit set was recorded weekly for 12 weeks after defoliation. Flowers and fruits were sampled at random every week, weighed, and frozen until analysis. For $\mathrm{CH}$ analysis, three replicates of 15 flowers/fruits per treatment and date were collected, frozen in liquid nitrogen, stored at $-28^{\circ} \mathrm{C}$, and then lyophilized. For gene expression analysis, three replicates of 10 flowers/fruits per treatment and date were collected, frozen in liquid nitrogen and stored as powder at $-80^{\circ} \mathrm{C}$. Woody samples (branches and new shoots) were harvested in control and defoliated branches at fruit set (i.e. twenty days after anthesis), and were prepared for $\mathrm{CH}$ analysis as described below.

\subsection{Carbohydrates extraction and analysis}

The procedure used for $\mathrm{CH}$ determination is that described in our recent studies [10, 11]. Briefly, $100 \mathrm{mg}$ of powdered samples were extracted with $1 \mathrm{ml}$ of $800 \mathrm{ml} \mathrm{l}^{-1}$ ethanol and purified sequentially using cation and anion exchange columns. The eluates were then passed through a C18 Sep-Pak cartridge (Waters-Millipore, Billerica, MA) and analyzed in a Spectra high performance liquid chromatography (HPLC) System (Spectra, San Jose, CA) equipped with a vacuum pump (Spectra P2000, Spectra) and a 
differential refractometer (Spectra R150, Spectra). Sucrose, glucose and fructose were identified according to their retention times. Results were expressed as milligram per gram dry weight (DW). Starch levels were determined in the pellets remaining after the extraction of soluble sugars. The residue was dissolved in $5 \mathrm{ml}$ water, gelatinized by autoclaving for 2 hours at $130^{\circ} \mathrm{C}$, centrifuged for $10 \mathrm{~min}$ and $4 \mathrm{ml}$ of supernatant removed. The sample was incubated by shaking for $2 \mathrm{~h}$ at $55^{\circ} \mathrm{C}$ with $0.1 \mathrm{ml}$ fucose (internal standard) (Sigma Chemica Co. Inc., Sigma-Aldrich Chemie Gmbh, Steinheim, Germany), $0.5 \mathrm{ml}$ of sodium acetate (pH 4.5) and $1 \mathrm{ml}$ of $1218 \mathrm{U}$ amyloglucosidase from Rhizopus (Sigma Chemica Co. Inc.). Samples were centrifuged for $30 \mathrm{~min}$ and $27000 \mathrm{~g}$, and the supernatant was dried in vacuo and redissolved in $1 \mathrm{ml}$ water. The glucose released was determined by HPLC as above. Results were expressed as milligram glucose released per gram DW. Three independent biological samples under each experimental condition were evaluated in technical triplicates

\subsection{Gene expression analysis by $q R T-P C R$}

RNA isolation, quantification, and quality analysis were performed as described in a recent work [29]. Total RNA was extracted from frozen tissues and subsequently treated with DNase I (RNase-Free DNase Set, Qiagen, USA). The amount of RNA was measured by spectrophotometric analysis (NanoDrop ND-1000 spectrophotometer, Thermo Fisher, USA). The absence of DNA contamination was checked by performing a no-reverse transcription assay which consisted of a PCR with each RNA sample using the Citrus actin primers. No amplified products were detected, which confirmed the purity of the RNA extracts. The transcripts present in $1 \mu \mathrm{g}$ of total RNA were reversetranscribed using the QuantiTect ${ }^{\circledR}$ Reverse Transcription Kit (Qiagen, USA) in a total volume of $20 \mu \mathrm{l}$. A $2.5 \mu \mathrm{l}$ aliquot of a 4-time diluted first-strand cDNA was used for each amplification reaction. Quantitative real-time PCR was carried out on a Rotor Gene Q 5-Plex (Qiagen, USA) using the QuantiTect ${ }^{\circledR}$ SYBR ${ }^{\circledR}$ Green PCR Kit (Qiagen, USA). The reaction mix and conditions followed the manufacturer's instructions with certain modifications. The PCR mix contained $2.5 \mu \mathrm{l}$ of diluted cDNA, $12.5 \mu \mathrm{l}$ of QuantiTect ${ }^{\circledR}$ SYBR Green PCR Master Mix (Qiagen, USA), $1.5 \mu$ of $0.3 \mu \mathrm{M}$ primer F, and $1.5 \mu \mathrm{l}$ of $0.3 \mu \mathrm{M}$ primer $\mathrm{R}$, the final volume being $25 \mu \mathrm{l}$. The cycling protocol for the amplification consisted of $15 \mathrm{~min}$ at $95^{\circ} \mathrm{C}$ for pre-incubation, then 40 cycles of $15 \mathrm{~s}$ 
at $94^{\circ} \mathrm{C}$ for denaturation, $30 \mathrm{~s}$ at $60^{\circ} \mathrm{C}$ for annealing and $30 \mathrm{~s}$ at $72^{\circ} \mathrm{C}$ for extension. RTPCR reactions were repeated three times for each gene and monitored in real time with the Rotor Gene Detector. After amplification, melting-curve analysis excluded artefactual amplifications. The relative expression of RNA transcripts was quantified with the threshold cycle values $(\mathrm{Ct})$ obtained from each sample using the $2^{-\Delta \Delta \mathrm{Ct}}$ method. Expression levels were calculated relative to the constitutively expressed $\beta$-act gene [29]. The relative gene expression level is given by $2^{-\Delta \Delta}$ Ct. Normalization was performed to the lowest Ct value. Three independent biological samples under each experimental condition were evaluated in technical triplicates. Primers used for gene expression analysis are listed in Table S1.

\subsection{Statistical analysis}

Data were subjected to ANOVA analysis and Student's t-test for mean separation using STATGRAPHICS Centurion software. When required, percentages were arcsintransformed to homogenize the variance.

\section{Results}

\subsection{Fruit growth and abscission}

The ovary-to-fruit transition occurred between 1 and 2 weeks after anthesis, and the cell division stage of fruit growth lasted for 4-5 weeks (Figs. 1A-D). In our experiment, the preanthesis flower abscission did not occur, but the post-anthesis flower abscission reached almost $20 \%$ of the flowers initially formed, and the physiological fruitlet drop reached up to $80 \%$ in the control branches (Figs 1E, F), coinciding with the onset of the rapid fruit growth stage (Fig. 1A-D).

Surprisingly, leaf removal 40 days before anthesis did not significantly reduce ovary growth or abscission up to the onset of the cell division stage 28 days after anthesis (DAA) (Figs. 1A-D). Thus, the effect of defoliation was postponed for more than 2 months. At that time, the abscission rate was significantly increased during the physiological fruitlet drop (Fig. 1F) and 15 days later (i.e. 58 days after anthesis) all the fruitlets from defoliated branches (DEF) abscised (Fig. 1E). 


\subsection{Carbohydrate metabolism}

In the flowers of control branches, the relative expression of SPS (sucrose biosynthesis) was upregulated 7.3-fold from -40 to -20 DAA (Fig. 2A), this correlating with a significant increase in sucrose concentration (Fig. 2E). Then, SPS expression progressively downregulated becoming nil at fruit set (i.e. 17 DAA) (Fig. 2A). Sucrose hydrolysis in the cytosol, mediated by SUS1 and SUS3, significantly increased (3-fold from -40 DAA to anthesis), and remained almost constant during the following 6 weeks (Fig 2B, C). On the other hand, the relative expression of the cw-INV, which hydrolyzes sucrose in the apoplast, increased 2-fold at the preanthesis, and remained almost constant until fruit set (+17 DAA), and then downregulated 40 DAA (Fig 2D). The increase in the expression of the enzymes related with sucrose hydrolysis correlated with a decrease in sucrose concentration from -20 DAA onwards (Fig. 2E). Glucose concentration significantly increased along with flower/ovary development (Fig. 2F) whereas fructose concentration decreased (Fig. 2G). Hexoses (glucose + fructose) concentration stayed almost constant until fruit set, when it increased due to the glucose contribution (Fig 2. H).

Defoliation significantly reduced ovary sucrose concentration at anthesis (Fig 2E), although SPS relative expression (sucrose biosynthesis) was constantly higher compared to the control flowers (Fig 2A). Given that sucrose hydrolysis was not significantly enhanced in flowers from defoliated branches until +17 DAA, the reduction in ovary sucrose concentration at anthesis might be due to the lack of transport from the leaves, i.e. to leaf removal. Consequently, the concentration of glucose and fructose also dropped (Fig. 2F-H).

Regarding starch metabolism in the control flowers, the relative expression of $A D P G P$ (starch biosynthesis) increased 1.3-fold from -40 to 0 DAA, and it was significantly downregulated after fruit set (Fig 3A). On the other hand, the relative expression of $\alpha$ $A M Y$ (starch hydrolysis) upregulated 7.5-fold at -20 DAA compared to -40 DAA, and also at fruit set (+17 DAA) (3.3-fold), after a temporary downregulation at anthesis (Fig. 3B). As a result, starch ovary concentration fell significantly from -20 DAA to fruit set (Fig. 3C). Surprisingly, defoliation 40 days before anthesis did not reduce significantly compared to the control starch concentration until fruit set, i.e. 57 days later, just before the increase in fruitlet growth rate (Fig. 1C, D), and due to a significant upregulation (almost 30-fold) in $\alpha$-AMY expression (Fig. 3B). The lack of an effect of 
defoliation on starch concentration, during the period from preanthesis to anthesis, might be explained by a simultaneous reduction in both starch synthesis (ADPGP expression) and hydrolysis ( $\alpha$-AMY expression) (Fig. 3B).

\subsection{Activation of energy depletion signaling and carbon fixation}

The process of flower development in control branches activated both the energy depletion signaling system, i.e. $\beta S n R K 1$ expression, and also the carbon fixation by means of Rubisco. In particular, we selected to analyze the small subunit of Rubisco $(R b c S$ ) because it is de novo synthesized in the nucleus and transported to the chloroplast to fix carbon [30]. Thus, in ovaries from control branches, $\beta S n R K 1$ expression was upregulated 2.4-fold -20 DAA and 3-fold at anthesis compared to -40 DAA, whereas $R b c S$ upregulated 3-fold during anthesis and fruit set (+17 DAA). At that time, $\beta$ SnRK1 was dowregulated. In the fruits from DEF branches, both mechanisms were enhanced and activated earlier (Fig 4). Moreover, fruits from DEF branches were able to mobilize $\mathrm{CH}$ from the bark tissue of young and mature branches, as seen by the analysis of glucose, fructose, sucrose and starch concentrations 20 DAA, which was significantly reduced compared to the control branches (Fig 5).

\section{Discussion}

At anthesis, Citrus accumulates starch in active chloroplasts and amyloplasts of the ovary walls. By contrast, during the ovary-to-fruit transition starch content progressively diminishes $[10,11]$ to satisfy the energy demanded by ovary cell division [31]. In fact, below a glucose threshold level, the SnRK1 energy sensor is upregulated and the development program is inhibited [23]. Surprisingly, defoliation of old and young leaves 40 days before anthesis did not reduce the starch content significantly in the ovary until fruit set +17 DAA (Fig. 3), i.e. two months later, whereas that of sucrose was significantly reduced during anthesis and fruit set (Fig. 2), in accordance with previous studies [10].

Our results suggest that the ovary regulates both starch biosynthesis and hydrolysis, depending on its sink demand and carbon availability, and this occurs through both the heterotrophic (sink organ) and autotrophic (source organ) routes of starch metabolism. 
In the heterotrophic route, $\mathrm{CH}$ needed for starch synthesis is provided by the hydrolysis of sucrose translocated from the leaves, a reaction mediated by sucrose synthase (SUS) which converts sucrose into UDP-glucose and ADP-glucose [16,17]. In our experiments, SUS1 and SUS3 expression significantly increased together with ADPGD expression (starch biosynthesis) during preanthesis (Fig. 2). Moreover, defoliation significantly reduced sucrose content in the ovary from -20 DAA (Fig. 2) with concomitant levels of $A D P G P$ expression (Fig. 3). In the autotrophic route, starch and sucrose synthesis in the leaves is coordinated through a feed-back-feed-forward mechanism which depends on tree sink demand [32]. In this process, ADPGP and SPS are the key enzymes regulating starch and sucrose synthesis, respectively $[16,17,19]$. According to our experiments, this self-regulatory mechanism can be also found in the flower, because in the flowers from control branches, $A D P G P$ expression was upregulated and SPS was downregulated at anthesis compared to -20 DAA, whereas in the flowers from DEF branches they were dowregulated and upregulated, respectively (Figs. 2, 3). The reduced sucrose concentration in the ovary due to defoliation, might explain this contrasting behavior. But the most surprising finding was the activation of 1) $\mathrm{CH}$ starvation signaling through upregulation of $\beta S n R K 1$ and 2) $\mathrm{C}$ fixation through upregulation of $R b c S$ in flowers from both control and DEF branches (Fig. 4). During the ovary-to-fruit transition, ovary growth rate is significantly increased due to mesocarp cell division [29] and therefore sink demand. However, the sink-to-source transition of young leaves occurs around 4 weeks after full bloom, and, since they cannot sustain the onset of ovary growth, abscission starts [8]. In control branches, we found a maximum increase in $\beta S n R K 1$ expression at anthesis, suggesting a $\mathrm{CH}$ deficit signaling [23]. Then, the ovary responded: 1) upregulating $R b c S$ expression (C fixation), and 2) maintaining $A D P G P$ (starch synthesis) and increasing $\alpha A M Y$ (starch hydrolysis) expression from anthesis to fruit set, which 3) resulted in a significant increase in glucose concentration at fruit set, and 4) afterwards, $\beta S n R K 1$ dowregulated in the ovary. These results are supported by the behavior of the ovaries on DEF branches, in which $\beta S n R K 1$ upregulation and $R b c S$ were significantly enhanced with respect to control branches. In early stages of development, the citrus exocarp presents stomata and photosynthetic capacity [33, 34], and transports C fixed in the form of sucrose to the mesocarp during cell division [33], an ability that decreases as the ovary grows [34]. Ovaries from DEF branches also increased $\alpha A M Y$ expression (starch 
hydrolysis) and $c w I N V$ expression (sucrose hydrolysis from the apoplast), suggesting a coordinated activation of several mechanisms to obtain $\mathrm{C}$ due to the limitation of $\mathrm{CH}$ supply imposed by defoliation [10].

Ovary growth and flower abscission rates did not differ significantly between fruitlets from control and DEF branches during the +20 DAA, differences starting during the physiological fruit drop (Fig. 1). The concentration of glucose in the developing ovaries of control branches increased significantly during fruit set, which is related to the high contribution generated by the hydrolysis of starch and sucrose. But the concentration of glucose in the developing ovaries of defoliated branches dropped significantly with respect to the control ovaries, and remained constant at $25 \mathrm{mg} \mathrm{g}^{-1} \mathrm{DW}$ (Fig. 2), which is similar to previous studies conducted with the same variety [11]. This suggests that there is a glucose threshold value that allows for the initial development of the ovary, regulated by $\mathrm{CH}$ signaling $[10,35]$. The concentration of glucose in a given tissue can vary depending on [39]: 1) hydrolysis of sucrose; 2) hydrolysis of starch; 3) $\mathrm{CO}_{2}$ fixation and glucose synthesis; 4) conversion to glucose-6-P by hexokinase to meet the respiratory demand. At the onset of the rapid growth stage fruits do not fix C [34], but hydrolyze sucrose in an extremely active manner. Ovaries from DEF branches that overcame fruit set managed to mobilize the reserves of the wood and hydrolyzed the sucrose, as shown by the reduced content of $\mathrm{CH}$ in the bark and the increased $c w$-INV expression. Similar results were reported by [10]. However, in fruits from control branches, it is worth noting the reduction in $c w-I N V$ gene expression and the upregulation of SUS1 and SUS3 genes during fruit set. A possible explanation would be the different products of the reaction: cw-INV generates glucose and fructose for respiration, while SUS generates fructose and also ADP-glucose, a precursor of cellulose which is needed to form new cell walls because the cell division $[17,19]$. Defoliation also reduced the expression of SUS1 in the ovary significantly, suggesting a main role of SUS1 compared to SUS3. The restriction of the availability and transport of sucrose to the ovary probably reduced the discharge through the symplast, which would diminish the content of sucrose in the cytosol and, hence, the expression of SUS1 [19].

\section{Conclusion}

In conclusion, the ability of flowers/fruitltes to mobilize $\mathrm{CH}$ reserves will determine their success during the physiological fruitlet drop and, therefore, crop load. 
Citrus flowers are able to accumulate starch in the ovary during ontogeny through a double mechanism: 1) their photosynthetic capacity (autotrophic route) and 2) the mobilization of reserves (heterotrophic route). The ovary upregulates the photosynthetic route when the $\mathrm{CH}$ disponibility is diminished, which is pointed out throught $\beta S n R K 1$ uprregulation. In accordance with our results, the role of starch in the Citrus ovary is to establish a glucose threshold level that allows the ovary-to-fruit transition developmental program (fruit set), regardless of the presence of leaves, until young leaves are photo-synthetically active and become source organs.

\section{References}

[1] S. Sutherland, Why hermaphroditic plants produce many more flowers than fruits: experimental tests with Agave mckelveyana, Evolution 41(1987) 750-759.

[2] M. Sawicki, E. Aït Barka, C. Clément, N. Vaillant-Gaveau, C. Jacquard, Crosstalk between environmental stresses and plant metabolism during reproductive organ abscission, J. Exp. Bot. 66 (2015) 1707-1719.

[3] J.L. Guardiola, F. García-Marí, M. Agustí, Competition and fruit set in the Washington navel orange, Physiol. Plant. 62 (1984) 297-302.

[4] J. Rodrigo, J. I. Hormaza, M. Herrero, Ovary starch reserves and flower development in apricot (Prunus armeniaca), Physiol. Plant. 108 (2000) 35-41.

[5] F. Bangerth, Abscission and thinning of young fruit and thier regulation by plant hormones and bioregulators, Plant Growth Regul. 31(2000) 43-59.

[6] E. Fadón, M. Herrero, J. Rodrigo, Dormant flower buds actively accumulate starch over winter in sweet cherry, Front. Plant Sci. 9 (2018)171.

[7] D.J. Iglesias, F.R. Tadeo, E. Primo-Millo, M. Talon, Fruit set dependence on carbohydrate availability in citrus tres, Tree Physiol. 23 (2003) 199-204.

[8] F. Rivas, A. Gravina, M. Agustí, Girdling effects on fruit set and quantum yield efficiency of PSII in two Citrus cultivars, Tree Physiol. 27 (2007) 527-535.

[9] M.M. Blanke, J.P. Bower, Small fruit problem in citrus trees, Trees 5 (1991) 239-243.

[10] J. Mehouachi, D. Serna, S. Zaragoza, M. Agusti, M. Talon, E. PrimoMillo, Defoliation increases fruit abscission and reduces carbohydrate levels in developing fruits and woody tissues of Citrus unshiu, Plant Sci. 107 (1995) 189197. 
Primo-Millo, M. Agustí, Self-pollination and parthenocarpic ability in developing ovaries of self-incompatible Clementine mandarins (Citrus clementina), Physiol. Plant. 148 (2013) 87-96.

[12] M.L. Alcaraz, J.I. Hormaza, J. Rodrigo, Ovary starch reserves and pistil development in avocado (Persea americana), Physiol. Plant. 140 (2010) 395404.

[13] M.L. Alcaraz, J.I. Hormaza, J. Rodrigo, Pistil starch reserves at anthesis correlate with final flower fate in avocado (Persea americana), PloS one 8 (2013) e78467.

[14] L. Reale, C. Sgromo, L. Ederli, S. Pasqualini, F. Orlandi, M. Fornaciari, M., F. Ferranti, B. Romano, Morphological and cytological development and starch accumulation in hermaphrodite and staminate flowers of olive (Olea europaea L.), Sex. Plant Reprod., 22 (2009) 109-119.

[15] G.I. Moss, B.T. Steer, P.E. Kriedemann, The regulatory role of inflorescence leaves in fruit-setting by sweet orange (Citrus sinensis), Physiol. Plant. 27 (1972) 432-438.

[16] E. Baroja-Fernández, F.J. Muñoz, T. Saikusa, M. Rodríguez-López, T. Akazawa, J. Pozueta-Romero, Sucrose synthase catalyzes the de novo production of ADPglucose linked to starch biosynthesis in heterotrophic tissues of plants, Plant Cell Physiol. 44 (2003) 500-509.

[17] E. Baroja-Fernández, F.J. Muñoz, J. Li, A. Bahaji, G. Almagro, M. Montero, M., E. Etxeberria, M. Hidalgo, M.T. Sesma, J. Pozueta-Romero, Sucrose synthase activity in the sus1/sus2/sus3/sus4 Arabidopsis mutant is sufficient to support normal cellulose and starch production, Proc Nat. Acad. Sci. 109 (2012) 321-326.

[18] T. Ohdan, P.B. Jr. Francisco, T. Sawada, T. Hirose, T. Terao, H. Satoh, Y. Nakamura, Expression profiling of genes involved in starch synthesis in sink and source organs of rice, J. Exp. Bot. 56 (2005) 3229-3244.

[19] Y.L. Ruan, Sucrose metabolism: gateway to diverse carbon use and sugar signaling. Ann. Rev. Plant Biol. 65 (2014) 33-67.

[20] E.W. Pavel, T. M. DeJong, Seasonal $\mathrm{CO}_{2}$ exchange patterns of developing peach (Prunus persica) fruits in response to temperature, light and $\mathrm{CO}_{2}$ concentration, Physiol. Plant. 88 (1993) 322-330. 
[21] S.N. Vemmos, G.K. Goldwin, The photosynthetic activity of Cox's

Orange Pippin apple flowers in relation to fruit setting, Ann. Bot. 73 (1994) 385391.

[22] M. Sawicki, B. Courteaux, F. Rabenoelina, F. Baillieul, C. Clement, E.A. Barka, C. Jacquard N. Vaillant-Gaveau, (2017). Leaf vs. inflorescence: differences in photosynthetic activity of grapevine, Photosynthetica 55 (2017) 58-68.

[23] S. Hulsmans, M. Rodriguez, B. De Coninck, F. Rolland, The SnRK1 energy sensor in plant biotic interactions, Trends Plant Sci. 21 (2016) 648-661.

[24] M. Agustí, F. García-Marí, J.L. Guardiola, Gibberellic acid and fruit set in sweet orange, Sci. Hort. 17 (1982) 257-264.

[25] A. Gómez-Cadenas, J. Mehouachi, F.R. Tadeo, E. Primo-Millo, M. Talon, Hormonal regulation of fruitlet abscission induced by carbohydrate shortage in citrus, Planta 210 (2000) 636-643.

[26] B. Martínez-Alcántara, D.J. Iglesias, C. Reig, C. Mesejo, M. Agustí, E. Primo-Millo, Carbon utilization by fruit limits shoot growth in alternate-bearing citrus tres, J. Plant Physiol. 176 (2015) 108-117.

[27] D.J. Iglesias, F.R. Tadeo, E. Primo-Millo, M. Talon, Carbohydrate and ethylene levels related to fruitlet drop through abscission zone A in citrus, Trees 20 (2006) 348-355.

[28] D.G. Sprugel, T.M. Hinckley, W. Schaap, The theory and practice of branch autonomy, Ann. Rev. Ecol. System. 22 (1991) 309-334.

[29] C. Mesejo, R. Yuste, C. Reig, A. Martínez-Fuentes, D.J. Iglesias, N. Muñoz-Fambuena, E. Primo-Millo, M. Agustí, Gibberellin reactivates and maintains ovary-wall cell division causing fruit set in parthenocarpic Citrus species, Plant Sci. 247 (2016)13-24.

[30] J.O. Berry, P. Yerramsetty, A.M. Zielinski, C.M. Mure, Photosynthetic gene expression in higher plants, Photosynth Res. 117 (2013) 91-120.

[31] K. Hartig, E. Beck, Crosstalk between auxin, cytokinins, and sugars in the plant cell cycle, Plant Biol. 8 (2006) 389-396.

[32] M.J. Paul, T.K. Pellny, Carbon metabolite feedback regulation of leaf photosynthesis and development, J. Exp. Bot. 54 (2003) 539-547. 
Fruits. I. $\mathrm{C}^{14} \mathrm{O}_{2}$ Uptake by Young Oranges in Light and in Dark, Plant Physiol. 35(1960) 425.

[34] S. Hiratsuka, M. Suzuki, H. Nishimura, K. Nada, Fruit photosynthesis in Satsuma mandarin, Plant Sci. 241 (2015) 65-69.

[35] J. Lastdrager, J. Hanson, S. Smeekens, Sugar signals and the control of plant growth and development, J. Exp. Bot. 65 (2014) 799-807.

[36] A. Komatsu, T. Moriguchi, K. Koyama, M. Omura, T. Akihama, Analysis of sucrose synthase genes in citrus suggests different roles and phylogenetic relationships, J. Exp. Bot. 53 (2002) 61-71.

[37] M.Z. Islam, X.M. Hu, L.F. Jin, Y.Z. Liu, S.A. Peng, Genome-wide identification and expression profile analysis of citrus sucrose synthase genes: investigation of possible roles in the regulation of sugar accumulation, PLoS One 9 (2014) e113623.

[38] L. Shalom, S. Samuels, N. Zur, L. Shlizerman, A. Doron-Faigenboim, E. Blumwald, A. Sadka, Fruit load induces changes in global gene expression and in abscisic acid (ABA) and indole acetic acid (IAA) homeostasis in citrus buds. J. Exp. Bot. 65 (2014) 3029-3044.

[39] J.S. Amthor, Respiration and crop productivity, New York, Springer Verlag (1989)

\section{Caption to Figures}

Figure 1. Early fruit growth and abscission in control (CNT) and defoliated (DEF) branches of Citrus clementina cv. Marisol. Each value is the average of 25 fruits randomly sampled from trees (A-D), and the average abscission of 8 branches (E-F). Figures $1 \mathrm{~B}$ and $1 \mathrm{D}$ are the same data as $1 \mathrm{~A}$ and $1 \mathrm{C}$, respectively, with a reduced axis scale. Standard errors are given as vertical bars. * indicates significant differences.

Figure 2. The time-course of the gene expressions of SPS, involved in sucrose synthesis (A) and SUS1, SUS3 and cwINV involved in sucrose hydrolysis (B-D), and sucrose (E), glucose $(F)$, fructose $(G)$ and glucose + fructore (hexoses, $H$ ) concentrations in the ovary of flowers from control (CNT) and defoliated (DEF) branches of Citrus 
clementina cv. Marisol. Each value is the average of three biological replicates. Standard errors are given as vertical bars. *indicates significant differences.

Figure 3. The time-course of the gene expressions of ADPGP involved in starch synthesis (A) and $\alpha A M Y$ involved in starch hydrolysis (B), and starch concentration (C) in the ovary of flowers from control (CNT) and defoliated (DEF) branches of Citrus clementina cv. Marisol. Each value is the average of three biological replicates. Standard errors are given as vertical bars. *indicates significant differences.

Figure 4. The time-course of the gene expression of $\beta S n R K 1$ involved in the $\mathrm{CH}$ starvation signaling (A) and the small subunit of Rubisco $(R b c S)(B)$ in the ovary of flowers from control (CNT) and defoliated (DEF) branches of Citrus clementina cv. Marisol. Each value is the average of three biological replicates. Standard errors are given as vertical bars. *indicates significant differences.

Figure 5. Effect of leaf removal on $\mathrm{CH}$ starvation in the bark tissue of branches and current shoots of control (CNT) and defoliated branches (DEF) of Citrus clementina cv. Marisol. Each value is the average of three biological replicates. Standard errors are given as vertical bars. *indicates significant differences. 
